\title{
Pulsed Thermography Signal Reconstruction Using Linear Support Vector Regression.
}

\author{
J. FLEURET ${ }^{1,2}$, S. EBRAHIMI ${ }^{1,2}$, X. MALDAGUE $^{1,2}$
}

\author{
${ }^{1}$ Faculty of Sciences and Engineering, University Laval, Quebec City, Quebec, Canada, \\ julien.fleuret.1@univ.laval.ca \\ ${ }^{2}$ Laboratory of Computer Vision and Numerical Systems, Multipolar Infrared Vision team, Quebec City, \\ Quebec, Canada
}

\section{Abstract}

This study introduces and evaluates a new approach for the reconstruction of image sequences acquired during non destructive testing by pulsed thermography. The proposed method consists in applying two linear support vector regressions, to model the evolution of the data from both a spatial and temporal point of view. Each regressor will map the data with respectively the number of pixels and the number of frames using a convex optimization. Then the regressors are used to predict a more robust representation of the data which is thus used to reconstruct the sequence. The proposed method has been applied to data related to a reference sample of carbon reinforced fiber with known defects. This approach has given good results in terms of noise reduction as well as the quality of the reconstructed image than most of the state of the art methods. The proposed method was able to outperform many of the state of the art algorithms and give comparable results otherwise despite being sensitive to non-uniform heating.

Keywords- Pulsed Thermography, Support Vector Regression, PT, SVR, materials, defect detection, IRNDT, active thermography

\section{Introduction}

Pulsed Thermography is a very popular non-destructive testing method for material inspection. This method consists in stimulating a sample using a short heating pulse. The pulse energy will be converted into heat and will spread through the material. Density variation in the material, cracks, inserts, void, air, any alteration or local non-uniformity will influence the diffusion and be registered by a thermal camera. The popularity of this method comes from its relatively low cost, and its ability to handle many different kinds of materials and defects. However thermal cameras are sensitive to a wide range of noises which will affect many pixels randomly when they do not create random patterns. For this reason the field of PT is eager to developt methods that can improve the detection of defects in the samples. Shepard et al. [1] introduced the Thermographic Signal Reconstruction (TSR), a method that is able to reconstruct the signal with significantely less noise. Such reduction of the noise allows the detection of deeper defects. The TSR consists in modeling the behavior of a sample subjet to a heat-pulse as a linear regression of the data into a natural logarithmic space. Due to the features of the samples and the defects as well as the frequency of acquisition, the relationship between the data in the logarithmic might be quasi-linear rather than linear which may create a reconstruction error. To correct this issue as well as offering an easier way to find defects in sequences, Shepard et al. have also proposed a method to obtain time derivatives from the reconstructed sequence. The TSR is still nowadays a very popular method which has been improved [2-6] and even adapted to different kinds of applications [7-9]. However one must note that it is a model based approach, which means it is based on an assumption. Lopez et al. $[10,11]$ have proposed a statistical based approach, which means that the signal is reconstructed from its statistics without any prior. The approach of Lopez et al. is name Partial Least Squares Thermography (PLST). The Partial Least Squares (PLS) regression is able to model the distribution of the data both spatially and temporally. The PLS during the regression decomposes both the data as well as their time representation into two sets of latent variables which are later minimized. The size of the latent space must be defined in advance. Also in order to highlight the location of the defects in the image the result of the PLS regression must be tricked. Nevertheless the PLST is able to highlight the location of the defects through the time, making them easier to segment than when the TSR is used.

To the best of our knowledge there is no other statistical approaches than the PLST in order to reconstruct and highlight defects in a raw thermogram. Support Vector Regression (SVR) has been used in many applications 
to predict representative and robust features learned from noisy features sets. Among the most famous applications of the SVR is the Bag of Visual Words. The SVR is able to manage a high dimensionaly features space and is independent from the input space. It is also known to be robust to noise [12]. This paper is structured as follows. In the next section, the Support Vector Regression is briefly introduced. Section 3 describes the proposed method. Section 4 explain how to apply the SVR to PT data. Section 5 details the experiments that have been conducted. Section 6 introduces the results that are discussed in section 7 . Section 8 concludes this work.

\section{Support Vector Regression}

This section outlines the mathematical basis of the Support Vector Regression (SVR) $[13,14]$. The SVR is a tool that has initially been introduced with another algorithm, the Support Vector Machine (SVM). The SVM is a statistical learning algorithm which has the ability to map an input domain $\mathcal{X}$ with a feature space $\mathcal{F}, \mathcal{X} \rightarrow \mathcal{F}$. The SVR makes a linear approximation in an output space $\mathcal{Y}$ of an unknown function. This is done by using non linear functions which allow to have linear modeling of the unknown regression. Let us consider a training set composed of $N$ input-output pairs as shown in equation (1)

$$
\Omega=\left\{\left(\mathbf{x}_{1}, y_{1}\right),\left(\mathbf{x}_{2}, y_{2}\right), \cdots,\left(\mathbf{x}_{N}, y_{N}\right)\right\}
$$

where $\mathbf{x}_{i} \in \mathcal{X}$ and $y_{i} \in \mathcal{Y}$ The expression of the function $f: \mathbf{x} \rightarrow y$ can be formulated as a convex optimization problem:

$$
\begin{array}{ll}
\underset{\mathbf{w}, b, \xi_{i}^{-}, \xi_{i}^{+}}{\operatorname{minimize}} & \frac{1}{2}\|\mathbf{w}\|^{2}+C \sum_{i=1}^{N}\left(\xi_{i}^{+}+\xi_{i}^{-}\right) \\
\text {subject to } & \left(\left\langle\mathbf{w}, \phi\left(\mathbf{x}_{i}\right\rangle+\mathbf{b}\right)-y_{i} \leq \epsilon+\xi_{i}^{+}\right. \\
& y_{i}-\left(\left\langle\mathbf{w}, \phi\left(\mathbf{x}_{i}\right\rangle+b\right) \leq \epsilon+\xi_{i}^{-}\right. \\
& \xi_{i}^{+}, \xi_{i}^{-} \geq 0
\end{array}
$$

Where $\forall i \in[1, N]$ and $\mathbf{w} \in \mathcal{F} . \xi_{i}^{+}$and $\xi_{i}^{-}$vectors correspond to the upper and lower error parameter in which the function $g(\mathbf{x})=\left\langle\mathbf{w}, \phi\left(\mathbf{x}_{i}\right\rangle+b\right.$ is allowed to deviate. The deviation is only allowed under the constraints that $[\epsilon, C] \geq \mathbf{0}^{T}$, where $\epsilon$ is a preset error and $C$ is a cost function. The two first constraints represent respectively the upper and lower boundaries. Note that in equation equation (2) any error smaller than $\epsilon$ does not require that $\xi_{i}^{+}$or $\xi_{i}^{-}$be non negative, hence it is not part of the cost function.

It is possible to formulate equation (2) as a kernel-based regression involving the Lagrange multiplier as shown in equation (3).

$$
\begin{array}{ll}
\underset{\alpha^{+}, \alpha^{-}}{\operatorname{maximize}} & -\frac{1}{2} \sum_{i, k}\left\{\left(\alpha_{i}^{+}-\alpha_{i}^{-}\right)\left(\alpha_{k}^{+}-\alpha_{k}^{-}\right)\right\} \\
& -\epsilon \sum_{i}\left(\alpha_{i}^{+}+\alpha_{i}^{-}\right)+\sum_{i}\left(\alpha_{i}^{+}-\alpha_{i}^{-}\right) \\
\text {subject to } & \sum_{i}\left(\alpha_{i}^{+}-\alpha_{i}^{-}\right)=0 \\
& 0 \leq \alpha_{i}^{ \pm} \leq C
\end{array}
$$

In this case the regression cost function is $g(\mathbf{x})=\sum_{i}\left(\alpha_{i}^{+}-\alpha_{i}^{-}\right) K\left(\mathbf{x}, \mathbf{x}_{i}\right)+b$ where $K\left(\mathbf{x}, \mathbf{x}_{i}\right)=\left\langle\phi(\mathbf{x}), \phi\left(\mathbf{x}_{i}\right)\right\rangle$ is a kernel function.

The interest of using a kernel-based regression rather than a direct regression, it is because it reduces the computational complexity. In the case of a direct regression a mapping with high dimensional data is needed which might be computationlly challenging. It is possible to express the kernel function by a matrix, which means that the mapping $\Phi: \mathbb{X} \rightarrow \mathbb{F}$ becomes a collection of dot products. From a computational point of view this property reduces the complexity. Note that the mapping function $\Phi$ can be unknown. In this case the kernel function can be replaced by a metric between two vectors. 
$15^{\text {th }}$ Quantitative InfraRed Thermography Conference, 6 - 10 July 2020, Porto, Portugal

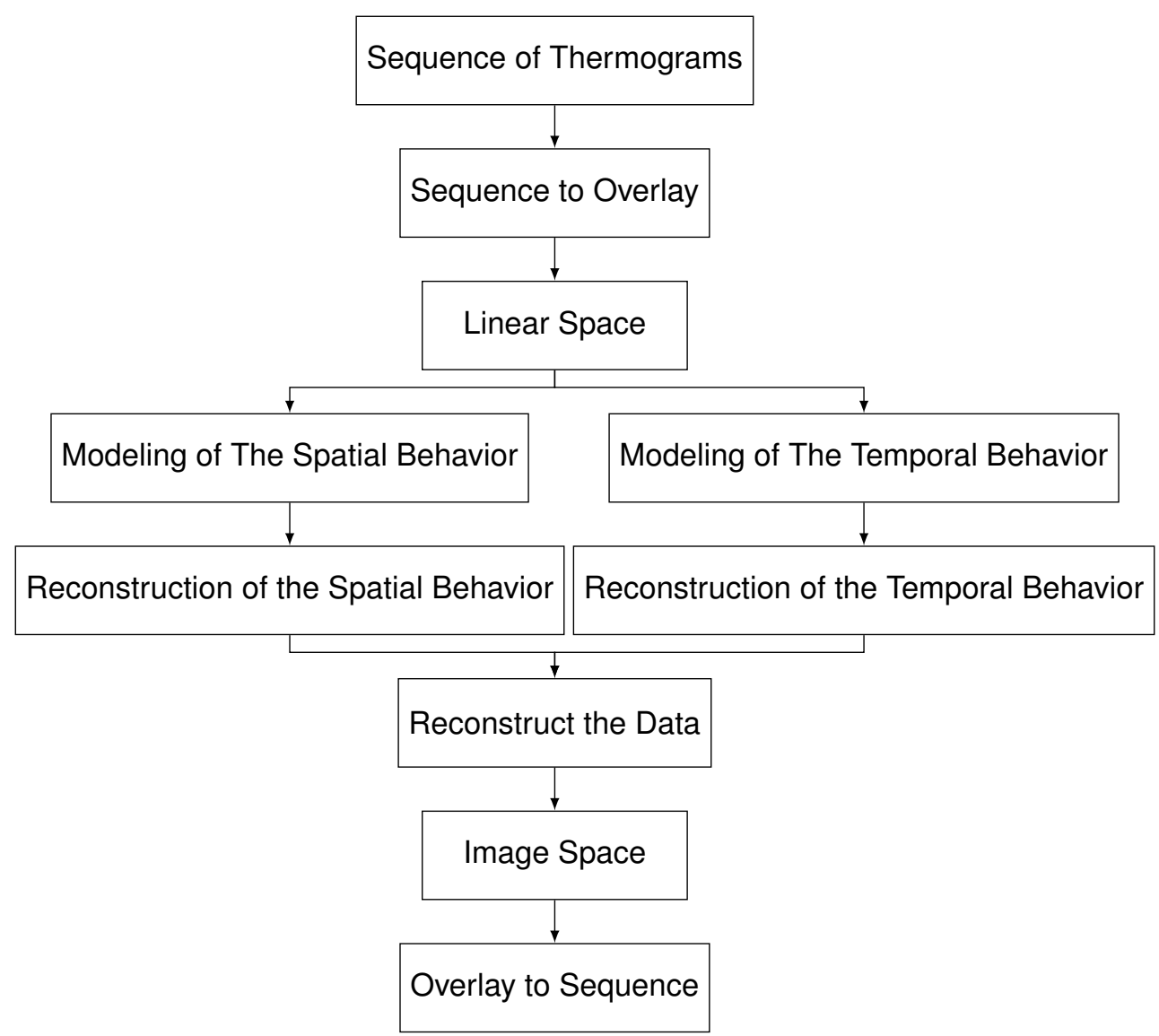

Figure 1: Proposed data reconstruction

\section{Application of the SVR to NDT}

In the field of pulsed thermography it is well known that the thermograms are sensitive to a wide range of noise. Let $X$ be a thermogram, which can be represented as:

$$
X=\sum_{t \in \mathbf{T}} \sum_{x \in \mathbf{P}} \sum_{i \in \mathbf{N}_{t}} a_{t, x, i} \phi_{t, x, i}
$$

$a_{i}$ represents a coefficient that is related to the raw intensities while $\phi_{i}$ is a basis function. It can be expressed either as a transform or as an adaptative function. $\mathbf{T}, \mathbf{P}$ represent respectively the number of frames and the total number of pixels in each frame. $\mathbf{N}_{t}$ represents the number of noise sources that are going to influence the image. Note that this number varies randomly from frame to frame.

From equation (4) it is clear that the noise sources influence the data both spatially and temporally. This highlights the need to have a spatial and temporal representation of the data to efficiently represent the model. For this reason in order to efficiently reconstruct the data, it is important to model the representation of the data.

In pulsed thermography the energy decay of a semi-infinite sample submited to a Dirac heating pulse can be expressed as:

$$
T(t)=\frac{Q}{e \sqrt{\pi t}}
$$

where $Q$ represents the quantity of energy absorbed by the sample and $e=\sqrt{k \rho C}$ is the thermal emissivity of the material. By applying the natural logarithm on equation (5) it becomes:

$$
\log (T(t))=\log (Q)-\frac{1}{2}(\log (e)+\log (\pi t))
$$




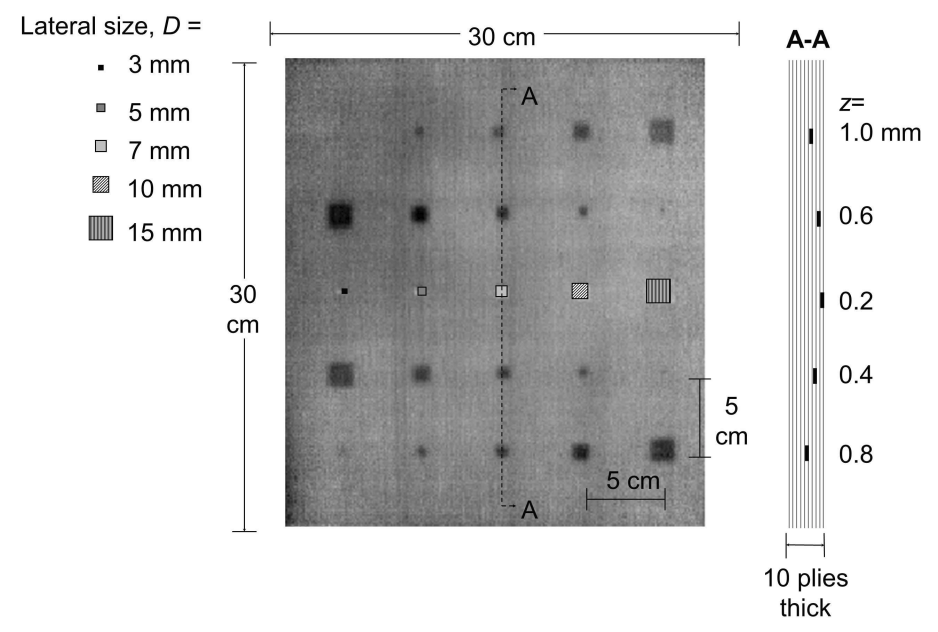

Figure 2: CFRP sample used for the experiments.

It is known that the emissivity of a material varies from material to material but is constant for a given material. The same observation can be made for the quantity of energy absorbed, it will vary depending of some experiemental parameters such as the distance between the light source and the target material, the power of the light sources, as well as the physical properties of the material; nevertheless as highlighted by equation equation (5), this variable does not depend on the time. Thus equation (6) can be expressed as

$$
\log (T(t))=\text { cst }_{1}-\frac{1}{2}\left(\text { cst }_{2}-\log (\pi t)\right)
$$

where cst $_{1}$ and $c s t_{2}$ are two constants. The linearity that the calculation of the logarithm offers is clear from equation (7). However this equation does not take into account the influence of the many noise sources. Shepard et al. [1] highlighted the influence of the noise sources, as well as the fact that the frequency of acquisition may mean that the linearity visible in equation (7) may not be respected anymore; nevertheless the signal will stay quasi-linear.

The originality of the proposed approach is to compute the a reconstruction based on the modeling of the temporal statistical distribution of each pixels as well as the model spatial distribution. The models of the spatial and temporal responses are computed from the linear representation of the data.

\section{Method}

The proposed approach has been compared with the state-of-the-art in terms of defect candidate detections i.e. TSR [1], PCT [15, 16], PPT [17] and PLST [10, 11]. We also compare several kernels as well as the implementation of the SVR. This section is structured in three parts. In the first part the metrics used are briefly introduced, then the case of study is detailed, the third part describes the protocol, and finally the performance of the proposed approach is compared with state-of-the-art method for each selected material.

\section{Case Study}

In order to evaluate the potential interest of the proposed method, the experiments were conducted on a reference Carbon Fiber Reinforced Plastic sample. This sample contains twenty-five Teflon inserts. These defects are divided into five batches of five defects. Each batch has five inserts having the same depth but different sizes (from $3 \times 3 \mathrm{~mm}^{2}$ to $15 \times 15 \mathrm{~mm}^{2}$ ). Also, each batch has been positioned at a specific depth (from 0.2 to $1 \mathrm{~mm}) 2$. 


\section{Protocol}

The materials have been evaluated under a classic pulse thermographic procedure. Each material has been stimulated from the front side by a pulse generated by two photographic flashes (Balcar FX 60, 5 ms thermal pulse, $6.4 \mathrm{~kJ} / \mathrm{flash})$. A mid-wave infrared (MWIR) camera FLIR Phoenix $(1.5 \mu \mathrm{m}$ to $5.0 \mu \mathrm{m}, 345 \mathrm{~Hz}, 14 \mathrm{bit}$ per pixel, $640 \times 512$ ) was used for data acquisition. For each experiment, a sequence of the 2000 frames was acquired during a period of 30 seconds. That duration ensured that both the warm-up and the cool-down period had been acquired by the sensor.

The first step consists in computing the cold image in order to only keep the part of the sequence related to the cooling. Then from this range, only the 400 first frames are kept. The number 400 is an empirical value, however during the tests reducing the sequence to 400 shows better results than processing the number 400 images must be adjusted depending on the frequency of acquisition. In the experiments conducted for this section 400 images was enough to ensure that the whole cooling period was included in the dataset. This also reduces the number of thermograms to process and with it the computational resources needed to process the data. Then each state of the art method was applied on the datasets. In the case of the TSR the first and second derivative are also compared.

\section{Metrics}

In order to assess the interest of the proposed approach and conduct a comparison the state of the art methods, two metrics have been selected. The proposed method offers two main interests; it is able to reconstruct the signal and to highlight subsurface defects. In order to assess the quality of the reconstruction, we choose to use the Signal over Noise Ratio (SNR). We use the formulation proposed by Usamentiaga et al. [18]

$$
S N R\left(\operatorname{roi}_{s}, \operatorname{roi}_{n}\right)=\frac{\left|\mu_{s}-\mu_{n}\right|}{\sqrt{\frac{\left(\sigma_{s}^{2}+\sigma_{n}^{2}\right)}{2}}}
$$

To evaluate the ability of each method to properly detect defects we use the accuracy metric. In addition to the

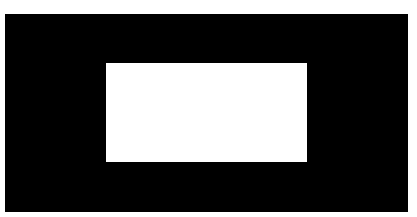

(a) ground truth

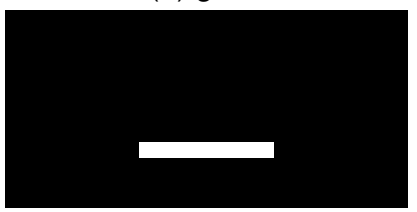

(d) false negative

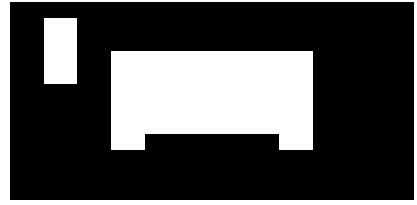

(b) result of a detection

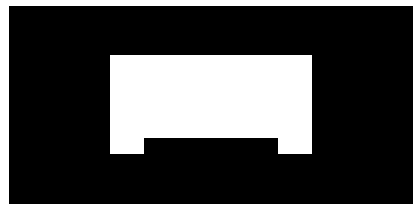

(e) true positive

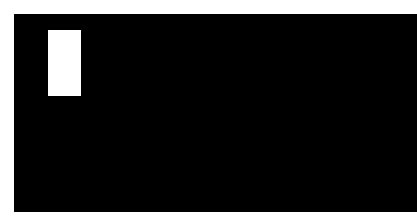

(c) false positive

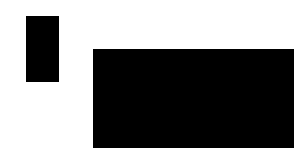

(f) true negative

Figure 3: Illustration of the different concepts used in Receiver Operating Characteristic approaches.

SNR, we use also the metric proposed by Shnayderman et al. [19]. This metric is sensitive to the distortion relative to a wide range of noises, while being quite simple to compute. This metric is computed in two steps. During the first step a distance is computed between the reference and the target image considering a regular grid. These values are then pooled using a summation in order to give the final score. The computation along a regular grid makes this metric more sensitive to the local variations. On each block of the grid the graphical measure is computed as shown in equation (9).

$$
D_{i, j}=\sqrt{\sum_{k=1}^{n}\left(s_{i, j, k}-\hat{s}_{i, j, k}\right)^{2}}
$$

In equation (9) $s_{i, j, k}$ and $\hat{s}_{i, j, k}$ represent respectively the $k^{t h}$ singular values of the reference and target image for the block located at the $i^{t h}$ row and $j^{\text {th }}$ column of the grid. Once the graphical measures have been com- 


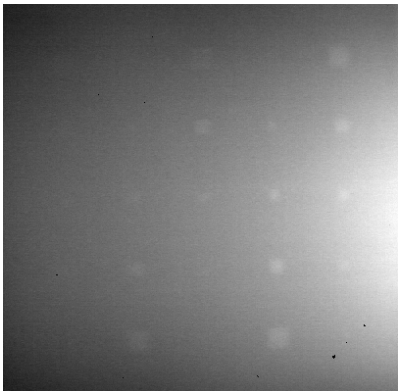

(a) Input

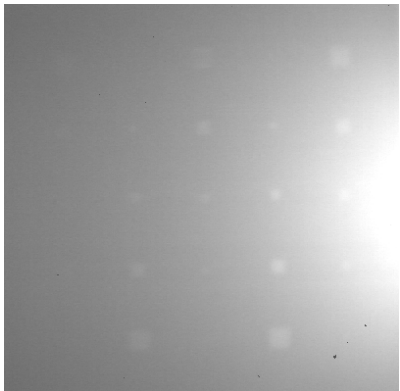

(b) TSR

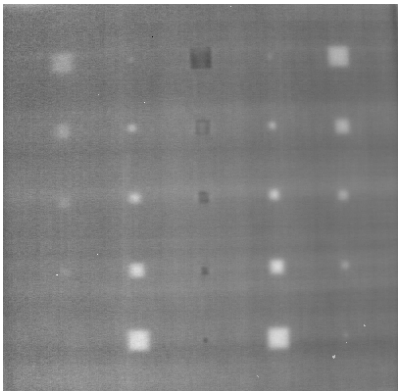

(c) PLST

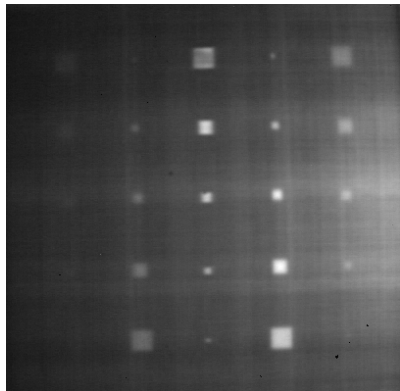

(d) SVR-Linear

Figure 4: Reconstructed images from differents approaches

puted the final score is calculated as the sum of the elements of the normalized absolute difference between the graphical measure and its median using equation (10).

$$
\text { score }=\frac{\sum_{i, j}\left|D_{i, j}-\operatorname{median}(D)\right|}{N}
$$

In equation (10) $N$ represents the number of blocks in the grid. Often if the reference and target image have $m$ rows and $n$ columns and the block size has a size of $b_{x} \times b_{y}$ then $N=\frac{m}{b_{y}} \cdot \frac{n}{b_{x}}$. Note that in the experiment we named this metric QSS (Quality Svd Score). In order to evaluate the ability of each method to accurately find the defects, we are using the Accuracy metric. The Accuracy comes from the Receiver Operating Characteristics, which have been used since the second world war to evaluate detections [20]. This metric is defined as:

$$
\text { Accuracy }=\frac{t p+t n}{t p+t n+f p+f n}
$$

where $t p$ i.e. true positive, is the number of pixels that have been properly labeled as part of the region of interest, $f p$ i.e. false positive, is the number of pixels that have been incorrectly labeled as part of a region of interest, $f n$ i.e. false negative, is the number pixels that have been incorrectly labeled as not part of the region of interest, and $t n$ i.e. true negative, pixels correctly labeled as not part of the region of interest. Figure 3 provides a visual aid to facilitate the understanding of these concepts.

\section{Results}

It has been observed during the processing that the sequence used has suffered from a random incident. In normal conditions the thermal pulse is generated by two flash lamps synchronized. In the sequence used it has been observed that the synchronization has not functioned properly and only the lamp on the left side has generate the pulse. This explain the lightness gradient visible in most of the images of figure 4 . Table 1 shows the metrics score calculated after processing the input sequence with both state of the art and the proposed methods. For PCT the metrics have been calculate on the images that offer the best contrasts among the first seven components. For the PPT, the image that offers the best contrasts was selected among a set of twenty images composed of the ten first and the ten last images. Unlike PCT and PPT, for the PLST as well as the SVR methods, the metrics score represents the average score computed on the whole sequence. Both SNR and QSS need sound areas in order to compute their score. The different noise sources varied a lot in the image from a both spatial and temporal point of view. In order to even increase the robustness of the metric, the scores are computed as the mean of each score computed between the area of the defects of interest and the area of 129 sound areas selected in the image. In order to help to interpret the SNRs score reported in table 1 have also been plotted in figure 5. The same images used to compute SNR and QSS score have also been used to compute the accuracy score table 2 . To compute the accuracy score, the results have been thresholded using the triangle algorithm [21]. In order to offer a more qualitative assessment of each reconstruction method, the results in figure 4 show the reconstruction for a randomly selected image of the input sequence. 
$15^{\text {th }}$ Quantitative InfraRed Thermography Conference, 6 - 10 July 2020, Porto, Portugal

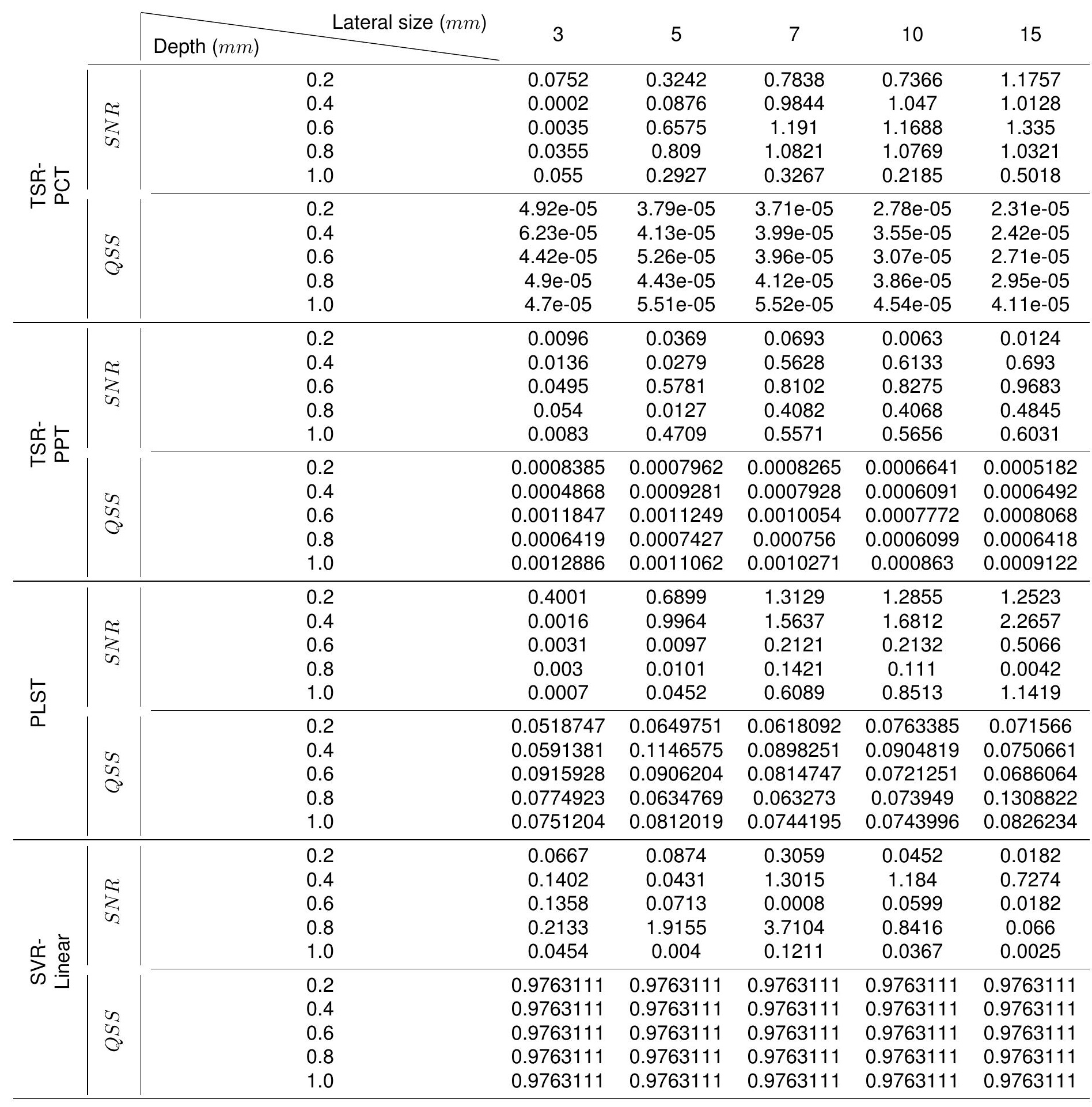

Table 1: SNR and QSS for state of the art methods.

\begin{tabular}{c|ccccc} 
& True Positive & True Negative & False Positive & False Negative & Accuracy \\
\hline TSR-PPT & 8 & 1 & 0 & 17 & 0.35 \\
TSR-PCT & 18 & 1 & 0 & 7 & 0.73 \\
PLST & 9 & 1 & 0 & 16 & 0.38 \\
Linear-SVR & 15 & 1 & 2 & 10 & 0.57
\end{tabular}

Table 2: Summary of the accuracy for each method. 
$15^{\text {th }}$ Quantitative InfraRed Thermography Conference, 6 - 10 July 2020, Porto, Portugal

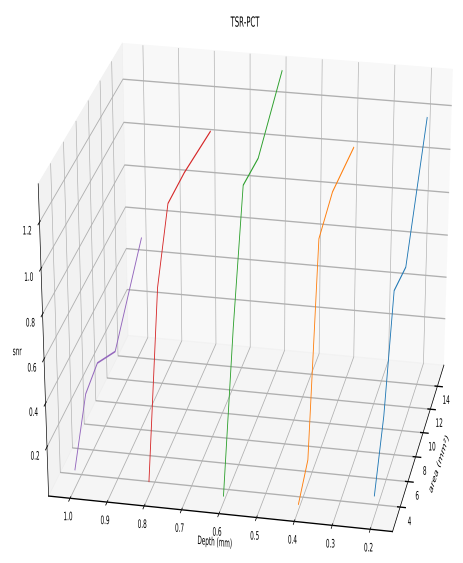

(a) TSR-PCT

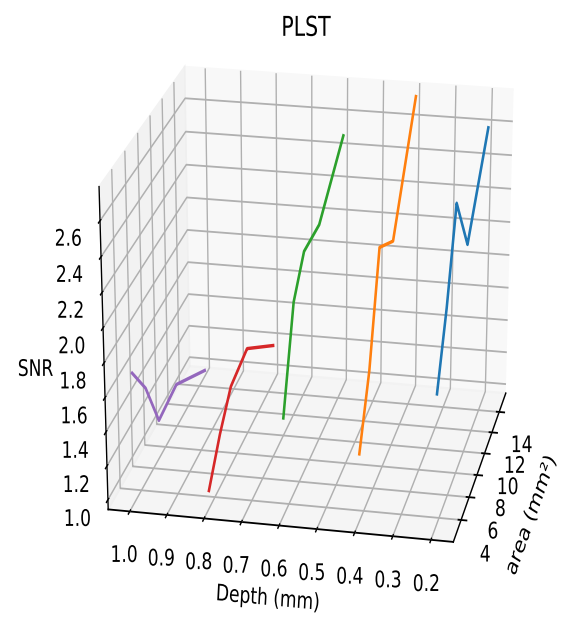

(c) PLST

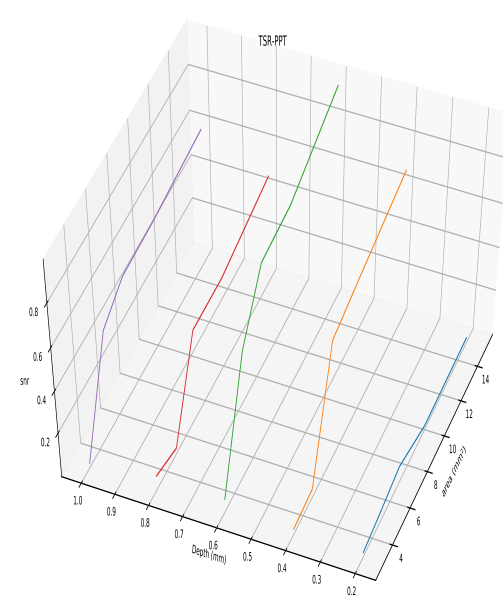

(b) TSR-PPT

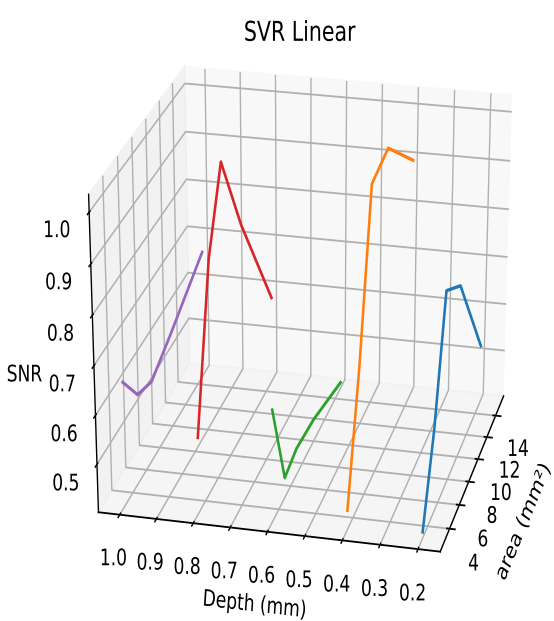

(d) SVR-Linear

Figure 5: Plot of the SNRs of the different methods 
From table 1 it is interesting to see the poor results that both TSR-PPT and TSR-PCT generate in terms of the QSS metric regarding their accuracy score. This highlights the fact that the signal reconstructed from the TSR, even if it is robust to most of the noises, still generates a relatively poor quality image. PLST and the linear SVR Regression provide significatively better results in terms of reconstructions. It is interesting to see that the reconstruction provided by the linear SVR has a constant QSS score for every defect. This means that the images reconstructed using the proposed method have a better image quality. Figure 4 also highlights that the proposed method is invarient to gradient inversion unlike other methods such as PLST. Nevertheless it is sensitive to non-uniform heating, like TSR. Only the PLST offers a robustness to non-uniform heating while being sensitive to gradient reversal.

The SNR values computed the TSR + PCT association and show the influence of the defect area on the score. An important gap on the scores is visible between the defects with an $7 \mathrm{~mm}^{2}$ and $10 \mathrm{~mm}^{2}$ for almost all inserts except those located at $1 \mathrm{~mm}$ from the surface. The SNR score for the inserts located at a depth of $1 \mathrm{~mm}$ from the surface seems at first not to follow the same trend as observed for the insert located at the other depths. This is most likely related to the non-uniform heating. The SNR score computed for TSR + PPT confirms the observation made for the method TSR + PCT. One can observe what seems to be a group of outlier variables located at $0.2 \mathrm{~mm}$ from the front surface. It is well known that PPT is not robust to gradient inversion. The image used to compute the score might have been taken closely before or after a gradient inversion which explains such poor results at this particular location. The SNR scores computed for the PLST follows mostly the trend observed with the previous approaches for the defects located between $0.2 \mathrm{~mm}$ and $0.6 \mathrm{~mm}$ below the surface. However the scores for the inserts located respectively at $0.8 \mathrm{~mm}$ and $1 \mathrm{~mm}$ shows a trend of declining regarding the area of the defect. Regarding the position of the defects on the sample, this behaviour is likely to be a consequence of the non-uniform heating.

The SNR scores for the SVR-Linear approach highlight the non-uniform heating. They show greater values for the defects located at $0.2 \mathrm{~mm}, 0.4 \mathrm{~mm}$ and $0.8 \mathrm{~mm}$ from the surface which represents the defects located the closest to the heating point. It is however surprising to see that the values for the defects located at $0.6 \mathrm{~mm}$ below the surface are significatively lower than those located at $1 \mathrm{~mm}$. Another trend is also visible from the defects located at $0.2 \mathrm{~mm}, 0.4 \mathrm{~mm}$ and $0.8 \mathrm{~mm}$. The SNR score increases for the defect area below $10 \mathrm{~mm}^{2}$ and then decreases. This may be related to the reconstructed image. In figure 4 it is clear that most of the $15 \mathrm{~mm}^{2}$ defects have lower lightness in comparison to defects located more in the center of the sample.

The accuracy score has been computed in order to assess the interest of the proposed approach for defect detection and segmentation, in table 2. PLST reconstructed sequences offers a significatively better SNR compared with TSR-PPT. However both do not allow the detection of half of the defects. For TSR-PPT the main reason is related to the reconstruction of the signal as well as the non-uniform heating. For PLST it is likely related to the non-robustness of gradient inversion which creates a wider histogram which thus will influence the result of the triangle method. TSR-PCT and the proposed Linear-SVR provide the best results: more than half of the defects segmented. The TSR-PCT seems to have been less influenced by the non-uniform heating than the proposed method. Regarding the previous results, it is likely that with a better sequence the results regarding the segmentation could also be better. However the results of this study regarding the proposed method are quite promissing with respect to both the quality of the reconstructed signal as well as the possibility to segment defects from the signal.

\section{Conclusion}

This paper introduces a new approach to reconstruct thermographic data acquired using pulsed thermography. This work models the behavior of the signal both spatially and temporally before reconstructing it. It is based on a support vector regression. The literature concerning the support vector regression, offers several implementations has well as several kernels, but in this study only the linear kernel has been used. The experiments conducted shows that the proposed method outperforms the state of the art regarding both its ability to reconstruction of the signal as well as the detection that can be done using automatic thresholding approaches. The proposed approach has been shown to be robust to many sources of noise; it is sensitive to non-uniform illumination. Future work includes the use of other kernels and implementation of the SVR on several types of material. 


\section{Acknowledgement}

The authors would like to thank Annette Schwerdtfeger for the time and energy she spent reviewing this article. This research was supported by the Fonds Quebecois de Recherche - Nature et Technologie (FQRNT) and the Natural Sciences and Engineering Research Council of Canada (NSERCC).

\section{References}

[1] Steven M Shepard. Advances in pulsed thermography. In Thermosense XXIII, volume 4360, pages 511516. International Society for Optics and Photonics, 2001.

[2] Steven M Shepard and Maria Frendberg Beemer. Advances in thermographic signal reconstruction. In Thermosense: Thermal Infrared Applications XXXVII, volume 9485, page 94850R. International Society for Optics and Photonics, 2015.

[3] Maria Frendberg Beemer and Steven M Shepard. Aspect ratio considerations for flat bottom hole defects in active thermography. Quantitative InfraRed Thermography Journal, 15(1):1-16, 2018.

[4] Steven M Shepard, James R Lhota, Bruce A Rubadeux, David Wang, and Tasdiq Ahmed. Reconstruction and enhancement of active thermographic image sequences. Optical Engineering, 42(5):1337-1343, 2003.

[5] SM Shepard, JR Lhota, and T Ahmed. Flash thermography contrast model based on ir camera noise characteristics. Nondestructive Testing and Evaluation, 22(2-3):113-126, 2007.

[6] Daniel L Balageas. Defense and illustration of time-resolved pulsed thermography for nde. In Thermosense: Thermal Infrared Applications XXXIII, volume 8013, page 80130V. International Society for Optics and Photonics, 2011.

[7] Daniel L Balageas, Jean-Michel Roche, François-Henri Leroy, Wei-Min Liu, and Alexander M Gorbach. The thermographic signal reconstruction method: A powerful tool for the enhancement of transient thermographic images. Biocybernetics and biomedical engineering, 35(1):1-9, 2015.

[8] Mariusz Kaczmarek. Tsr method for burns investigation approach. 2018.

[9] Andrés David Restrepo-Girón and Humberto Loaiza-Correa. New advances in multidimensional processing for thermal image quality enhancement. In Recent Advances in Applied Thermal Imaging for Industrial Applications, pages 202-248. IGI Global, 2017.

[10] Fernando Lopez, Vicente Nicolau, Xavier Maldague, and Clemente Ibarra-Castanedo. Multivariate infrared signal processing by partial least-squares thermography. In Proceedings of the 16th International Symposium on Applied Electromagnetics and Mechanics, Québec (Canada), 2013.

[11] Fernando Lopez, Clemente Ibarra-Castanedo, Vicente de Paulo Nicolau, and Xavier Maldague. Optimization of pulsed thermography inspection by partial least-squares regression. Ndt \& $E$ International, 66:128-138, 2014.

[12] Harris Drucker, Christopher JC Burges, Linda Kaufman, Alex J Smola, and Vladimir Vapnik. Support vector regression machines. In Advances in neural information processing systems, pages 155-161, 1997.

[13] Alex J Smola and Bernhard Schölkopf. A tutorial on support vector regression. Statistics and computing, 14(3):199-222, 2004.

[14] Karl S Ni and Truong Q Nguyen. Image superresolution using support vector regression. IEEE Transactions on Image Processing, 16(6):1596-1610, 2007.

[15] Nikolas Rajic. Principal component thermography. Technical report, Defence Science and Technology Organisation Victoria (Australia), 2002. 
$15^{\text {th }}$ Quantitative InfraRed Thermography Conference, 6 - 10 July 2020, Porto, Portugal

[16] Bardia Yousefi, Stefano Sfarra, Clemente Ibarra Castanedo, and Xavier PV Maldague. Comparative Analysis on Thermal Non-Destructive Testing Imagery Applying Candid Covariance-Free Incremental Principal Component Thermography (CCIPCT). Infrared Physics \& Technology, 85:163-169, 2017.

[17] Xavier Maldague and Sergio Marinetti. Pulse phase infrared thermography. Journal of applied physics, 79(5):2694-2698, 1996.

[18] R Usamentiaga, Clemente Ibarra-Castanedo, and X Maldague. More than fifty shades of grey: Quantitative characterization of defects and interpretation using snr and cnr. Journal of Nondestructive Evaluation, 37(2):25, 2018.

[19] Aleksandr Shnayderman, Alexander Gusev, and Ahmet M Eskicioglu. An svd-based grayscale image quality measure for local and global assessment. IEEE transactions on Image Processing, 15(2):422429, 2006.

[20] Marina Sokolova, Nathalie Japkowicz, and Stan Szpakowicz. Beyond Accuracy, F-score and ROC: a Family of Discriminant Measures for Performance Evaluation. In Australasian joint conference on artificial intelligence, pages 1015-1021. Springer, 2006.

[21] Gregory W Zack, William E Rogers, and Samuel A Latt. Automatic measurement of sister chromatid exchange frequency. Journal of Histochemistry \& Cytochemistry, 25(7):741-753, 1977. 